\title{
Aspects of topoi: Corrigenda and acknowledgements
}

\section{Peter Freyd}

"Aspects of Topoi" [1] appeared in July 1972 through the Herculean efforts of Bernard ${ }^{1}$ Neumann with the able assistance of Tim Brook and Max Kelly. The original manuscript was written in haste during the last two weeks of the author's visit to Australia. Somehow just five months later it appeared in print. Among the difficulties surmounted was a set of page proofs which having been sent via slow steamer by the U. of Penn. administration arrived after the paper was in press.

Tim Brook discovered the nonsense in the original manuscript about $\exists_{f}$ and, consequently, the proof therein of Lemma 2.615. As the creator of that nonsense I didn't see through it until it was so late that I was practically forced to telegraph a patch-up job. Though the page proofs didn't arrive on time, that did, and amazingly the patch-up got included. (Note that 2.615 is easy if we already know 3.32. The trick was to avoid 3.32 until after 3.31.$)$

Bill Mitchell in Chicago pointed out that I neglected to show that the $\Omega$-condition is essentially algebraic. (The fact is, $\$ 2$ was written before I recalled Lawvere telling me years ago that the axioms of categories are not just partial algebraic, that they are as I have now termed them, "essentially algebraic".) The domain of the characteristic map operator, namely the set of monomorphisms, is equationally defined as those maps $A \rightarrow B$ such that $x=y$ in the pullback $\begin{gathered}P \stackrel{x}{\longrightarrow} A \\ A \longrightarrow B \\ \downarrow\end{gathered}$

Received 14 August 1972.

1 Correct spelling: Bernhard. Editor. 
A much easier and better example than that of 2.71 exists for an unsolvable topos: let $T$ have as objects ordered 4-tuples $\left\langle A, s_{1}, s_{2}, a\right\rangle$ where $A$ is a set, $s_{1}, s_{2}$ automorphisms on $A$ and $a$ is a positive integer. A map from $\left\langle A, s_{1}, s_{2}, a\right\rangle$ to $\left(B, t_{1}, t_{2}, b\right)$ is any function $f: A \rightarrow B$ such that for some positive $n$,

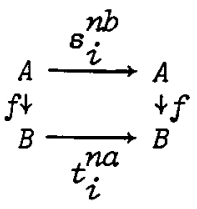

commutes for $i=1,2$. The forgetful functor is a logical morphism, $T$ is 2-valued, boolean, and its arithmetic is standard. Let $A_{n}$ be $Z_{n} \vee Z$, that is the set obtained by identifying the zero of the cyclic group of order $n$ with the zero of the infinite cyclic group. Let $s_{1}$ act as the shift operator $(m \rightarrow m+1)$ on the infinite cyclic part, the identity elsewhere. Let $s_{2}$ act as the rotation operator (again $m \rightarrow m+l)$ on the finite cyclic part, the identity elsewhere. Then $\mathrm{U}_{n}\left\langle A_{n}, s_{1}, s_{2}, 1\right\rangle$ fails to have a maximal well-pointed subobject.

Section 5.6 is all wrong. The error occurs earlier, namely the stupid assumption that a colimit of functors, each of which preserves epimorphic families, does the same. The lines about preserving epimorphic families in 3.21, through 3.24 should be struck. Fortunately we didn't use those lines until 5.6 where the results though false are answers to the right questions. A replacement for Section 5.6 appears below.

The paper shows a systematic - though unconscious - omission of the name of Alexander Grothendieck, for which I can only apologize and view with some wonderment. That I could have written, for example, an introduction in which I compared the development of the theories of abelian categories and topoi, without mentioning Grothendieck's dominating role in each, must be taken as evidence of an aberration stemming I suspect from a touch of mathematical chauvinism. 


\subsection{Some standard recoveries}

There exist bicomplete topoi, even boolean and 2-valued, with no exact functors into the category of sets. Nothing like a stalk functor. On the other hand, every countable exact sub-topos of any bicomplete topos is exactly embeddable in a power of the category of sets. Perhaps nothing better demonstrates the utility of the elementary version of topoi. The way in which one would likely use the existence of enough exact set-valued functors is to verify elementary assertions. Knowing that the countable sub-topoi allow enough exact set-valued functors is, of course, sufficient for this use.

We shall need the definitions of two special properties on topoi. Given a reflexive, symmetric relation $R \subset A \times A$ in a topos we may inductively define its powers, $R^{n+1}=R^{n} \circ R$, where $\circ$ means composition of relations and $R^{0}$ is the diagonal $A \stackrel{\Delta}{\longrightarrow} A \times A$. The countable union $U R^{\prime 2}$ need not exist. But if it does then it is an equivalence relation and the smallest equivalence relation containing $R$. If for every reflexive symmetric relation this countable union does exist we'll call the topos E-standard.

For arbitrary $R \subset A \times A$ we may consider the coequelizer $A \rightarrow F$ of $R \rightarrow A \times A \stackrel{p_{1}}{\longrightarrow} A, \quad R \rightarrow A \times A \stackrel{p_{2}}{\longrightarrow} A$, and the pullback $\begin{aligned} & E \rightarrow A \\ & \downarrow \\ & A \rightarrow F\end{aligned} . E \subset A \times A$ is, of course, an equivalence relation and using only the effectiveness of all equivalence relations, $E$ is easily seen to be the smallest equivalence relation containing $R$. E-standardness, therefore, says for reflexive symmetric $R$, that the just constructed $E$ is the union of the $\left\{R^{n}\right\}$.

For any $R \subset A \times A$ we can easily construct its "opposite", $R^{\text {op }}$ and define $\bar{R}=R \cup R^{\text {op }} \cup \Delta$ to obtain the smallest reflexive relation containing $R$. In an E-standard topos, $U \vec{R}^{n}$ is the smallest equivalence relation containing $R$. Note, and note well, that the construction of each $\vec{R}^{\prime}$ uses only the predicates of near-exactness:

$$
R^{\mathrm{op}}=\operatorname{Im}(R \rightarrow A \times A \stackrel{t}{\longrightarrow} A \times A)
$$


where $t$ is the twist map, $\vec{R}^{n} \circ \bar{R}=\operatorname{Im}\left(P \stackrel{\left\langle f_{2} g\right\rangle}{\longrightarrow} A \times A\right)$ where

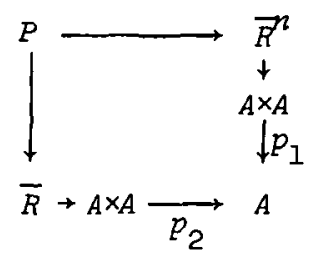

is a pullback, $f=\bar{R} \rightarrow A \times A \stackrel{p_{1}}{\longrightarrow} A, \quad g=\vec{R}^{n} \rightarrow A \times A \stackrel{p_{2}}{\longrightarrow} A$.

LEMMA 5.61 for topoi. If $T$ is E-stcondard, $T: T \rightarrow T^{\prime}$ a leftexact functor that preserves epimorphic families, then $T$ is exact.

Proof. If $T$ is left-exact and preserves epimorphic families, then it is near-exact (clearly such implies that $T$ preserves epimorphisms, and by applying to the empty family, preserves 0 ; moreover, $A \stackrel{u_{1}}{\longrightarrow} C$, $B \stackrel{u_{2}}{\longrightarrow} C$ display $C$ as $A+B$ iff the pullback of $u_{1}, u_{2}$ is 0 , $u_{1}, u_{2}$ each monomorphic and jointly epimorphic). We must show that $T$ preserves coequalizers. Given $x, y: B \rightarrow A$ let $R=\operatorname{Im}\left(M \stackrel{\left\langle x_{2} y\right\rangle}{\longrightarrow} A \times A\right)$. The coequalizer of $x, y$ is clearly the same as the coequalizer of $R \rightarrow A \times A \stackrel{p_{1}}{\longrightarrow} A, R \rightarrow A \times A \stackrel{P_{2}}{\longrightarrow} A$. Let $A \rightarrow F$ be such, $\begin{aligned} & E \rightarrow A \\ & \downarrow+\downarrow \\ & A \rightarrow F\end{aligned}$ a pullback, let $T A+F^{\prime}$ be a coequalizer of $T R \rightarrow T A \times T A \stackrel{P_{1}}{\longrightarrow} T A, \quad T R \rightarrow T A \times T A \stackrel{P_{2}}{\longrightarrow} T A$, and $\begin{aligned} & E^{\prime} \rightarrow T A \\ & \downarrow \\ & T A\end{aligned} F^{\prime}$ a pullback. $T A \rightarrow T F=T A \rightarrow E^{\prime}$ iff $T E=E^{\prime} . E^{\prime}$ is the smallest equivalence relation containing $T R . E=U \vec{R}^{n}$ and near-exactness implies $T\left(\vec{R}^{n}\right)=\overrightarrow{T R}^{n}$. If $T$ preserves epimorphic families then $T E=T\left(U \vec{R}^{n}\right)=U T\left(R^{n}\right)=\overrightarrow{W T}^{n}$, that is, $\overrightarrow{U R R}^{n}$ exists and hence it is the smallest equivalence relation containing $T R$. Hence $T E=E^{\prime}$.

We say that a topos is $N$-standard if it has a natural numbers object $N$ and the maps $1 \stackrel{n}{\longrightarrow} N$, through the standard natural numbers, form an 
epimorphic family. We will show that $N$-standard implies $E$-standard and in the presence of an NNO, E-standard implies $N$-standard.

A countably cocomplete topos is, of course, both E-standard and $N$-standard. Note that if $T \rightarrow T^{\prime}$ is an exact embedding, $T^{\prime}$, $E$-standard, then $T$ must be $E$-standard. Which two statements provide the easy half of

THEOREM 5.62. A small (boolean, 2-valued) topos may be exactly embedded in a bicomplete (boolean, 2-valued) topos iff it is E-standard.

Proof. Given any small topos $T$, let $S^{T^{\text {Op }}}$ be the category of contravariant set-valued functions, $T \rightarrow S^{T^{\text {op }}}$ the representation functor $A \rightarrow H_{A}$. There is a closure operator $j: \Omega \rightarrow \Omega$ in $S^{T^{\circ p}}$ which makes $T \rightarrow S^{T^{\mathrm{OP}}} \rightarrow S h_{j}$ preserve epimorphic families. Since $T \rightarrow S^{T^{\mathrm{p}}}$ and $S^{T^{\text {op }}}+S h_{j}$ are left-exact, Lemma 5.61 says for $T, E$-standard, that $T \rightarrow S h_{j}$ is exact.

The closure operator is just what it has to be. Given an $A$-crible $\left\{B_{i} \rightarrow A\right\}$ its closure in the set of all maps $C \rightarrow A$ such that the set of $\begin{array}{ll} & P_{i} \rightarrow C \\ \text { pullbacks } & \downarrow \\ & B_{i} \rightarrow A\end{array}$ yields an epimorphic family $\left\{P_{i} \rightarrow C\right\}$. (The closure must be at least this large because $\operatorname{UIm}\left(H_{B_{i}} \rightarrow H_{A}\right) \rightarrow \operatorname{UIm}\left(H_{C} \rightarrow A\right)$ must become an isomorphism in $S h_{j}$ if $T \rightarrow S h_{j}$ is to preserve epimorphic families.) To verify that we have described a closure operation requires a number of steps.

First, the closure is a crible. Given $C \rightarrow A$ in the closure of $P_{i}+C \quad P_{i}^{\prime}+C^{\prime}$ $\left\{B_{i} \rightarrow A\right\}$ let $C^{\prime} \rightarrow C$ be arbitrary, $\begin{aligned} & \downarrow \\ & B_{i} \rightarrow A\end{aligned} \underset{P_{i} \rightarrow C}{+}$ pullbacks. Then 
$P_{i}^{\prime} \rightarrow C^{\prime}$

$\downarrow_{i}+A$ is a pullback each $i$, and since $\left\{P_{i}+C\right\}$ is an epimorphic family, so is $\left\{P_{i}^{\prime} \rightarrow C^{\prime}\right\}$ (because $T / C \rightarrow T / C^{\prime}$ preserves same). The closure operation is clearly inflationary. To see that it is idempotent, let $\left\{C_{j} \rightarrow A\right\}$ be the closure of $\left\{B_{i} \rightarrow A\right\}$ and $D \rightarrow A$ in the closure of $\left\{C_{j} \rightarrow A\right\}$. We must show that $D \rightarrow A$ is in the closure of $\left\{B_{i} \rightarrow A\right\}$. Let $P_{i, j} \rightarrow C_{j}$

${ }_{i} \rightarrow \begin{aligned} & \downarrow \\ & i\end{aligned}$ be a pullback each $i, j$. For fixed $j,\left\{P_{i, j} \rightarrow C_{j}\right\}$ is an

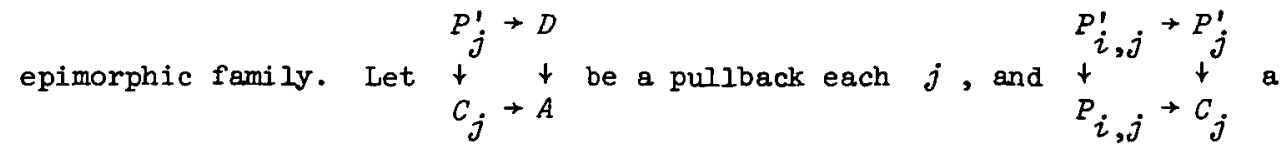
pullback each $i, j$. Because pullbacks of epimorphic families are again epimorphic, each $j,\left\{P_{i, j}^{\prime} \rightarrow P_{j}^{\prime}\right\}$ is epimorphic, hence $\left\{P_{i, j}^{\prime} \rightarrow D\right\}_{I \times_{J}}$ is $P_{i}^{\prime \prime}+D$

epimorphic. Now let $\begin{aligned} & \downarrow \\ & B_{i} \rightarrow A\end{aligned}$ be pullbacks. We wish to show that $\left\{P_{i}^{\prime \prime} \rightarrow D\right\}$ is epimorphic. But each $P_{i, j}^{\prime \prime} \rightarrow D$ factors through $P_{i}^{\prime \prime} \rightarrow 0$ regardless of $j$, and $\left\{P_{i, j}^{\prime} \rightarrow D\right\}$ epimorphic implies $\left\{P_{i}^{\prime \prime} \rightarrow D\right\}$ epimorphic.

For the naturality of the closure operations let $A^{\prime} \rightarrow A$ be

$$
B_{i}^{\prime}+A^{\prime}
$$

arbitrary, $\left\{B_{i} \rightarrow A\right\}$ an $A$-crible, $\underset{B_{i} \rightarrow A}{\downarrow}$ a pullback each $i$. First, suppose that $C \rightarrow A$ is in the closure of $\left\{B_{i} \rightarrow A\right\}$. Let $\begin{aligned} & C^{\prime} \rightarrow A^{\prime} \\ & C\end{aligned}$ be a pullback. We wish to show that $C^{\prime} \rightarrow A^{\prime}$ is in the closure of $\left\{B_{i}^{\prime}+A\right\}$. If $\begin{aligned} & P_{i} \rightarrow C \\ & B_{i} \rightarrow A\end{aligned}$ and $\begin{aligned} & P_{i}^{\prime} \rightarrow C^{\prime} \\ & P_{i} \rightarrow C\end{aligned}$ are pullbacks each $i$, then we obtain pullbacks $P_{i}^{\prime}+C^{\prime}$ $t_{i}^{\prime}+A^{\prime}$ and $\left\{P_{i}^{\prime} \rightarrow C^{\prime}\right\}$ being the pullback of an epimorphic family is 
again such that $C^{\prime} \rightarrow A^{\prime}$ is in the closure of $\left\{B_{i}^{\prime}+A^{\prime}\right\}$.

Second, if $C^{\prime} \rightarrow A^{\prime}$ is in the closure of $\left\{B_{i}^{\prime} \rightarrow A^{\prime}\right\}$ that is, from

$$
P_{i}^{\prime} \rightarrow C^{\prime}
$$

the pullbacks ${\underset{B}{i}}_{B_{i}^{\prime} \rightarrow A^{\prime}}^{+},\left\{P_{i}^{\prime} \rightarrow C^{\prime}\right\}$ is epimorphic then quite clearly

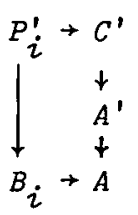

is a pullback each $i$, and $C^{\prime} \rightarrow A$ is in the closure of $\left\{B_{i} \rightarrow A\right\}$. Let $Q \rightarrow C^{\prime}$ $\downarrow+$ be a pullback. Then the original $C^{\prime} \rightarrow A^{\prime}$ is in the $A^{\prime}$-crible $A^{\prime} \rightarrow A$ generated by $Q \rightarrow A^{\prime}$, hence in the pullback of the closure of $\left\{B_{i} \rightarrow A\right\}$.

Finally we must show that the closure of an intersection is the intersection of the closures. Rather clearly the closure operation preserves order hence it suffices to prove, symbolically, $\vec{B} \cap \bar{B}^{\prime} \subset \overline{B \cap B^{\prime}}$, that is, if $C \rightarrow A$ is in the closures of both $\left\{B_{i} \rightarrow A\right\}$ and $\left\{B_{j}^{\prime} \rightarrow A\right\}$ then it is in the closure of $\left\{B_{i} \rightarrow A\right\} \cap\left\{B_{j}^{\prime} \rightarrow A\right\}$. Let $P_{i} \rightarrow C \quad P_{j}^{\prime} \rightarrow C \quad B_{i, j}^{\prime \prime} \rightarrow B_{j}^{\prime} \quad P_{i, j}^{\prime \prime} \rightarrow P_{j}^{\prime}$ $B_{i}^{+A}, \begin{aligned} & + \\ & B_{j}^{\prime} \rightarrow A\end{aligned} \begin{aligned} & +B_{i} \rightarrow A \\ & P_{i} \rightarrow C\end{aligned}$ be pullbacks each $i, j$. For fixed $j,\left\{P_{i, j}^{\prime \prime} \rightarrow P_{j}^{\prime}\right\}$ is epimorphic because $\left\{P_{i} \rightarrow C\right\}$ is and pullbacks of eximorphic families remain such. Because $\left\{P_{j}^{\prime} \rightarrow C\right\}$ is epimorphic, the somposite $\left\{P_{i, j}^{\prime \prime} \rightarrow C\right\}$ is epimorphic. But for each $i, j, \begin{aligned} & P_{i, j}^{\prime \prime} \rightarrow C \\ & B_{i, j}^{\prime \prime} \rightarrow A\end{aligned}$ is a pullback, $\left\{B_{i, j}^{\prime \prime} \rightarrow A\right\}$ is the intersection of $\left\{B_{i} \rightarrow A\right\}$ and $\left\{B_{j}^{\prime} \rightarrow A\right\}$ hence $C \rightarrow A$ is in the closure of the intersection.

We have therefore defined a closure operation. We must show that $T \rightarrow S^{T^{O p}} \rightarrow S h_{j}$ preserves epimorphic families. Given $\left\{B_{i} \rightarrow A\right\}$ epimorphic 
in $T$ we wish to show that $\left\{B_{B_{i}} \rightarrow{ }_{A}\right\}$ when reflected in $S h_{j}$ is epimorphlc, equivalently, that $\operatorname{UIm}\left(H_{B_{i}} \rightarrow H_{A}\right) \rightarrow H_{A}$ becomes an isomorphism. But $\operatorname{UIm}\left(H_{B_{i}}+H_{A}\right)$ corresponds to an $A$-crible whose closure is all of $H_{A}$ and that is precisely the condition that a subobject of ${ }_{A}$ becomes, upon reflection, the entire subobject.

$T \rightarrow S h_{j}$ is faithful, because being exact it suffices to show that for $A^{\prime} \rightarrow A$ not an isomorphism in $T$, that $H_{A^{\prime}} \rightarrow H_{A}$ does not become an isomorphism in $S h_{j}$. If it were to do so, then $A^{\prime} \rightarrow A$ must be in the closure of the $A$-crible generated by $A^{\prime} \rightarrow A$, which it clearly is not.

Note that given $B \leftrightarrow C \leftrightarrow A$ that $C \rightarrow A$ is in the closure of the $A$-crible generated by $B \rightarrow A$ and hence any closed $A$-crible is the closure of a family of monomorphisms. Given $\left\{B_{i}+A\right\}, C^{\prime} \rightarrow C$ is in the closure iff $C^{\prime}=U\left(B_{i} \cap C^{\prime}\right)$. We may thus identify the closed $A$-cribles with the ideals in $\operatorname{Sub}(A)$ closed under arbitrary union. If $\operatorname{Sub}(A)$ is boolean then any such ideal is closed under double negation and we see that if $T$ is boolean then so is $S h_{j}$.

COROLLARY 5.63 for topoi with NNO. E-standard implies N-standard.

Proof. If $T$ is $E$-standard with NNO we may exactly embed it in a bicomplete topos. Bicompleteness, indeed just countable cocompleteness, implies $N$-standardness. The invariance of NNO's under exact functors thus implies that $T$ is $N$-standard.

The critical lemma for much that follows:

LEMMA 5.64. A countable N-standard boolean topos may be logically mapped to a 2-valued $N$-standard boolean topos.

Proof. Let $B$ be a countable $N$-standard boolean topos. We seek an ultra-filter $F \subset B(1, \Omega)$ so that $B / F$ is still $N$-standard. In a 2-valued boolean topos, $N$-standardness is equivalent to the fact that for every $B \subset N, B \neq 0$ there exists a natural $n$ and a map $I \rightarrow B$ such 
that $1 \rightarrow B \rightarrow N=1 \stackrel{n}{\longrightarrow} N$. For any filter, $T: B \rightarrow B / F$ is $S$-full, that is, for any $A \in B$, $\operatorname{Sub}(A) \rightarrow \operatorname{Sub}(T A)$ is onto. Hence we seek an ultrefilter, $F$, such that for any $B \subset N$ in $B$ either there exists $U \in F$ such that $U \times B=0$, or there exists natural $n$ such that in the pullback $\begin{aligned} & P \rightarrow 1 \\ & +\rightarrow N \\ & B \rightarrow N\end{aligned}$, it is the case that $P \in F$.

Let $\left\{B_{n}\right\}_{n=1}^{\infty}$ be an enumeration of the subobjects of $N$. We construct a descending chain of subobjects of 1 as follows:

$U_{0}=1 ;$

given $U_{n}$ if $U_{n} \times B_{n+1}=0$ then set $U_{n+1}=U_{n}$, if $U_{n} \times B_{n+1} \neq 0$

let $m$ be the first natural number such that in the pullback

$\begin{gathered}P \\ U_{n}^{+} B_{n+1} \rightarrow \underset{N}{+m}\end{gathered}, P \neq 0$, and set $U_{n+1}=P$.

We note in the case $U_{n} \times B_{n+1} \neq 0$ that $U_{n+1} \rightarrow U_{n} \times B_{n+1}+U_{n} \times 1=U_{n}$, hence $U_{n+1} \subset U_{n}$. Hence there exists an ultra-filter $F$ containing all $U_{n}$, and that ultra-filter clearly has the desired property. (In fact, the set $F=\left\{V \subset 1 \mid \exists_{n}, U_{n} \subset V\right\}$ is already an ultra-filter.)

THEOREM 5.65. Every comtable N-standard (boolean) topos is exactly (Zogically) embeddable in a product of $N$-standard well-pointed topoi.

Proof. Theorem 2.65 is correct, namely every small topos may be exactly embedded in a boolean topos and the embedding can preserve epimorphic families. Hence an $\mathrm{N}$-standard topos can be exactly embedded in an $N$-standard boolean topos. If the given topos is furthermore countable then we may take a countable sub-model of the range to contain the image of the embedding, and we reduce the theorem to the boolean case.

An exact functor between boolean topoi is faithful iff it kills no objects. Given countable $N$-standard boolean $B$ and $A \in B$ then $B / A$ is again countable $N$-standard and boolean. If $B^{\prime}$ is well-pointed, $B / A \rightarrow B^{\prime}$ exact then $B \rightarrow B / A \rightarrow B^{\prime}$ does not kill $A$. Hence it suffices to show that every countable $N$-standard boolean topos may be logically 
mapped to an $N$-standard well-pointed topos. Lemma 5.64 says we may further reduce to the two-valued case. That is, we wish to show that any countable 2-valued $N$-standard boolean topos may be logically embedded into a $N$-standard well-pointed topos.

We will say that an object $A$ is pointed if there exists $1 \rightarrow A$. We recall that a boolean topos is well-pointed iff each non-zero object is pointed. Given any $A \in B, A \neq 0, B \rightarrow B / A$ moves $A$ to a pointed object. If $B$ were $N$-standard and boolean then so is $B / A$. If moreover, $B$ is countable, then we can apply Lema 5.64 to obtain a 2-valued $N$-standard and boolean $B^{\prime}$ and a logical map $B / A \rightarrow B^{\prime}$. Clearly $B^{\prime}$ can be cut down to a countable elementary submodel. If further, $B$ is 2-valued then the composite $B \rightarrow B^{\prime}$ being exact is faithful.

Hence given any countable 2-valued $N$-standard boolean topos $B$ and a non-zero $A \in B$ there exists a countable 2-valued $N$-standard boolean $B$ and a logical embedding $B \rightarrow B^{\prime}$ so that $A$ becomes pointed in $B^{\prime}$.

Let $\left\{A_{n}\right\}_{n=1}^{\infty}$ be an enumeration of the non-zero objects of $B$ and using the last paragraph define inductively, a sequence of countable 2-valued $N$-standard boolean topoi $\left\{B_{n}\right\}_{n=0}^{\infty}$, and logical embeddings $B_{n}+B_{n+1}$, with $B_{0}=B$, and such that $A_{n+1}$ becomes pointed in $B_{n+1}$. Let $\bar{B}$ be the colimit of the $B_{n}^{\prime} s$. We obtain a logical embedding $B \rightarrow \bar{B}$ and every non-zero object in $B$ becomes pointed in $\bar{B}$.

$\bar{B}$ is $N$-standard as follows: given $\bar{f}, \bar{g}: \bar{N}+\bar{B}$ in $\bar{B}, f \neq g$, there exists $n$ so that $B_{n} \rightarrow \bar{B}$ maps onto $\bar{f}, \bar{g}$. Let $f, g: N \rightarrow B$ in $B_{n}$ be ancestors. Because $B_{n}$ is $N$-standard there exists $1 \stackrel{m}{\longrightarrow} N$ in $B_{n}$ so that $1 \stackrel{m}{\rightarrow} N \stackrel{f}{\longrightarrow} B \neq 1 \stackrel{m}{\rightarrow} N \stackrel{g}{\rightarrow} B$. Because $B_{b} \rightarrow \bar{B}$ is faithful $\left(B_{n}\right.$ is 2-valued and $B_{n} \rightarrow B_{n}$ is exact) we retain the inequality in $\bar{B}$. We now iterate again to obtain a sequence $\left\{\bar{B}_{n}\right\}$, of 2-valued $N$-standard boolean topoi, $\bar{B}_{0}=B$, logical embeddings $\bar{B}_{n} \rightarrow \bar{B}_{n+1}$ which carry every non-zero object in $\bar{B}_{n}$ to a poisted object in $\bar{B}_{n+1}$. Again 
we take the colimit to obtain $\bar{B}_{w}$, again $\bar{B}_{w}$ is $N$-standard and boolean. But now $\bar{B}_{w}$ is well-pointed. Any non-zero $\bar{A} \in \bar{B}_{w}$ must have an ancestor $A$ in some $\bar{B}_{n}$, and $A$ becomes pointed in $\bar{B}_{n+1}$, hence remains so in $\bar{B}_{w}$.

COROLLARY 5.66. N-stondard implies E-standard.

Proof. As observed in 5.5 an $N$-standard well-pointed topos is $E$-standard. Hence every countable subtopos of an $\mathrm{N}$-standard topos is embeddable in an E-standard topos, and such is clearly enough to imply E-standardness.

THEOREM 5.67. A countable topos is exactly embeddable in a power of the category of sets iff it is E-standard.

Proof. By Lemma 5.61 a well-pointed topos is embeddable into sets by the function $(1,-)$. We note that Theorem 5.62 implies that any $E$-standard topos is exactly embeddable in an $N$-standard topos. Hence any $E$-standard topos can be embedded in a product of $\mathrm{N}$-standard well-pointed topoi, each in turn embeddable into sets.

THEOREM 5.68. An E-standard topos is exactly embeddable in a product of ultropowers of the category of sets.

Proof. First embed $T$ via Theorem 5.62 into an $N$-standard topos. Second, for any $x, y: A \rightarrow B, x \neq y$ specialize to an $N$-standard boolean topos so that $x, y$ are separated. Any elementary structure is embedded in an ultraproduct of its countable submodels. Each of those can be exactly mapped to the category of sets. Hence for each $x \neq y$ we obtain an exact functor to an ultrapower of sets that separates $x, y$.

THEOREM 5.69. There exists a boolean 2-valued $N$-standard topos of the power of the continum with no exact functors to the category of sets.

There exists a boolean 2-valued bicomplete topos with no exact functors to the category of sets.

Proof. Given a two-valued boolean topos $B$ and an object $A \in B$ we'll say that $A$ is measurable if there exists an ultra-filter $F \subset \operatorname{Sub}(A)$ closed under countable intersections. Suppose $B$ is bicomplete, $S: B \rightarrow B^{\prime}$ exact, $B^{\prime}$ well-pointed and $N$-standard. Then 
every object $A$ in $B$ is measurable as follows: choose $I \rightarrow S A$ and define $F \subset \operatorname{Sub}(A)$ as the set of subobjects $A^{\prime} \rightarrow A$ such that $1 \rightarrow S A$ factors through $S A^{\prime} \rightarrow S A$. $F$ is easily seen to be an ultra-filter. That an ultra-filter in a complete boolean algebra is closed under countable intersection is equivalent to the more often cited property that any countable partition of unity meets the ultra-filter, that is, given a sequence $\left\{A_{n}\right\}_{n=0}^{\infty}$ of pair-wise disjoint subobjects of $A$ such that $\mathrm{U}_{n}=A$ we must show that $A_{n} \in F$ for some $n$. Because $A \simeq \sum A_{n}$ there

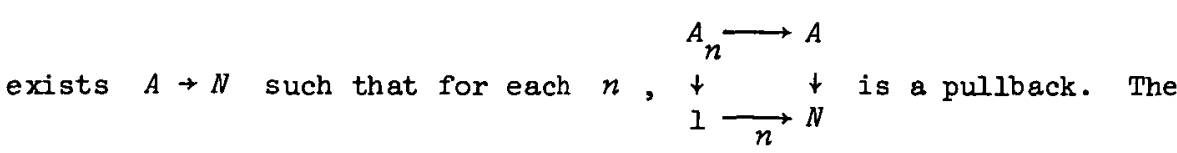
chosen $1 \rightarrow S A$ yields a natural number $m$ such that $1+S A+S N=1 \stackrel{m}{\rightarrow} N$ in $B^{\prime}$. And hence $A_{m} \in F$.

To show that a given bicomplete 2-valued boolean topos $B$ has no exact set-valued function it suffices to show that $B$ has an object not measurable.

If a boolean algebra is separable, that is if it has a countable dense subset, a subset being dense if below every non-zero element there is a non-zero element in the subset, then any ultra-filter closed under countable intersection is easily seen to be principal, thus an atomless separable boolean algebra has no such ultra-filter. We first construct a 2-valued $N$-standard boolean topos which yields an atomless boolean algebra.

For any group $G$ and strictly descending sequence of subgroups $\left\{G_{n}\right\}$ the topos $\lim _{\rightarrow} S^{n}$ is an example of such. We can be more explicit by taking $G$ to be the group of integers and instead of a sequence, taking the directed set of non-zero subgroups. The resulting category $B_{1}$ may be directly described as having ordered triples $(A, s, a)$ as objects, where $A$ is a set, $s$ is an automorphism on $A$ and $a$ is a positive integer. A map from $(A, s, a)$ to $(B, t, b)$ is a function $f: A \rightarrow A^{\prime}$ such that for some positive integer $n$, 
$A \stackrel{s^{n b}}{\longrightarrow} A$

${ }_{B} \underset{t^{n a}}{\longrightarrow}{ }_{B}^{\downarrow f}$ commutes. The forgetful functor to sets is a logical

morphism, which fact tells one how to prove that $B_{1}$ is a topos. The object $\langle z, s, 1\rangle, z$ the group of integers, $s(x)=x+1$, contains no atoms.

Let $B_{2}$ be a countable elementary submodel of $B_{1}$. The existence of an object without atoms is elementary, hence let $A \in B_{2}$ be such that $\mathrm{Sub}(A)$ has no atoms. Using Theorem 5.62 let $B_{3}$ be a bicomplete topos, $T: B_{2} \rightarrow B_{3}$ an exact functor that preserves epimorphic families, the second condition insuring that $T$ embeds $\operatorname{Sub}(A)$ as a dense subalgebra of $\mathrm{Sub}(T A)$. Sub(TA) is therefore atomless and separable and $B_{3}$ can have no set-valued exact functors.

To get down to the power of the continuum we note first that every subobject of $T A$ is a union of subobjects coming from $B_{2}$, hence $\operatorname{Sub}(T A)$ is of the power of the continuum. Any map $T A \rightarrow N$ is distinguished by the

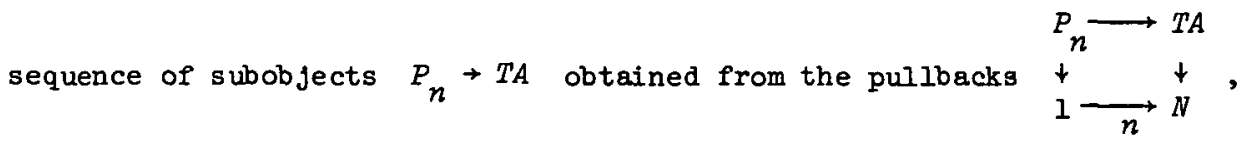
hence $B(T A, N)$ is of the power of the continuum. Let $B_{4}$ be an elementary submodel of $B_{3}$ such that $B_{3}(T A, N)=B_{4}(T A, N)$ and such that $B_{4}$ is of the power of the continuum. The existence of exact $S: B_{4}+B^{\prime}$ where $B^{\prime}$ is well-pointed and $N$-standard still implies that $T A$ is measurable (for the proof we used only the existence of enough maps from $T A$ to $N$, namely one to describe every countable partition). $\operatorname{Sub}(T A)$ is still separable and atomless, hence there is no such $S$. 


\section{Reference}

[1] Peter Freyd, "Aspects of topoi", Buzl. Austral. Math. Soc. 7 (1972), $1-76$.

Department of Mathematics,

University of Pennsylvania,

Philadelphia,

Pennsylvania, USA. 